\title{
Oclusão duodenal após cirurgia da aorta abdominal: relato de caso
}

\author{
Duodenal obstruction following abdominal aortic surgery: \\ case report
}

\author{
Adenauer Marinho de Oliveira Góes Junior ${ }^{1}$, \\ Alexandre Petnys ${ }^{2}$, Edgar Rabboni ${ }^{2}$, Milton Alves das Neves Junior ${ }^{1}$, Paulo Henrique Petterle ${ }^{3}$, \\ Rafael Couto Melo ${ }^{1}$, Tatiana Rocha Protta ${ }^{1}$, Weverton Terci ${ }^{3}$
}

\section{Resumo}

A maior parte dos artigos sobre obstrução duodenal após cirurgia aórtica cita dados referentes às correções da doença aneurismática e não da doença aterosclerótica. Não obstante, é consenso que se trata de uma complicação rara, cuja incidência é menor do que $1 \%$. Os autores relatam o caso de um paciente submetido a enxerto aorto-bifemoral que apresentou, como complicação pós-operatória, oclusão duodenal. O paciente foi tratado com reintervenção cirúrgica e uso de remendo de grande omento para síntese do retroperitônio. A revisão da literatura indica que a maioria dos casos responde bem ao tratamento conservador, e a conduta cirúrgica normalmente só é necessária quando aderências são a causa da obstrução ou quando o tratamento clínico não é satisfatório após 2 semanas.

Palavras-chave: Oclusão, duodeno, cirurgia, aorta.

\section{Introdução}

A incidência de complicações gastrointestinais após cirurgia de aorta varia de 6,6 a 21\%. Essas complicações são associadas a risco aumentado de morbidade e mortalidade e incluem mais freqüentemente íleo paralítico, hemorragia digestiva alta, enterocolite por Clostridium difficile, isquemia de cólon, pancreatite e colecistite aguda $^{1}$.

Obstrução duodenal é uma complicação incomum após cirurgia da aorta abdominal, ocorrendo em menos de $1 \%$ dos pacientes ${ }^{2}$. A maioria dos casos descritos na

\begin{abstract}
Most articles on duodenal obstruction following aortic surgery report data relative to repairs of aneurysmal disease, not atherosclerotic disease. However, duodenal obstruction is an uncommon complication, occurring in less than $1 \%$ of patients. We report a case of a patient submitted to aortobifemoral bypass reconstruction who had duodenal obstruction as postoperative complication. The patient was treated with surgical intervention and omental patching for retroperitoneal synthesis. Literature review indicates that most cases respond well to the conservative treatment, and surgical conduct is usually only required when adherences are causing the obstruction or when clinical treatment is not satisfactory after 2 weeks.
\end{abstract}

Keywords: Occlusion, duodenum, surgery, aorta.

literatura inclui pacientes tratados para doença aneurismática. Devido à raridade dessa complicação, o diagnóstico é freqüentemente retardado, o que resulta em significativa morbidade.

\section{Relato do caso}

Paciente masculino de 55 anos procurou o ambulatório de cirurgia vascular com queixa de claudicação de membros inferiores para distância menor do que 50 metros, incluindo musculatura do quadril. Relatava também disfunção erétil.

Referia antecedentes de tabagismo e etilismo de longa data. Negava outras comorbidades, mas referia história

1. Médico. Residente, Serviço de Cirurgia Vascular, Hospital do Servidor Público Municipal de São Paulo (HSPM), São Paulo, SP.

2. Médico(a). Assistente, Serviço de Cirurgia Vascular, HSPM, São Paulo, SP.

3. Cirurgião vascular. Ex-residente, Serviço de Cirurgia Vascular, HSPM, São Paulo, SP.

Trabalho realizado no Serviço de Cirurgia Vascular, Hospital do Servidor Público Municipal de São Paulo (HSPM), São Paulo, SP.

Não foram declarados conflitos de interesse associados à publicação deste artigo.

Artigo submetido em 20.05.08, aceito em 08.09.08.

J Vasc Bras. 2008;7(4):384-388.

(C) 2008 by Sociedade Brasileira de Angiologia e de Cirurgia Vascular 


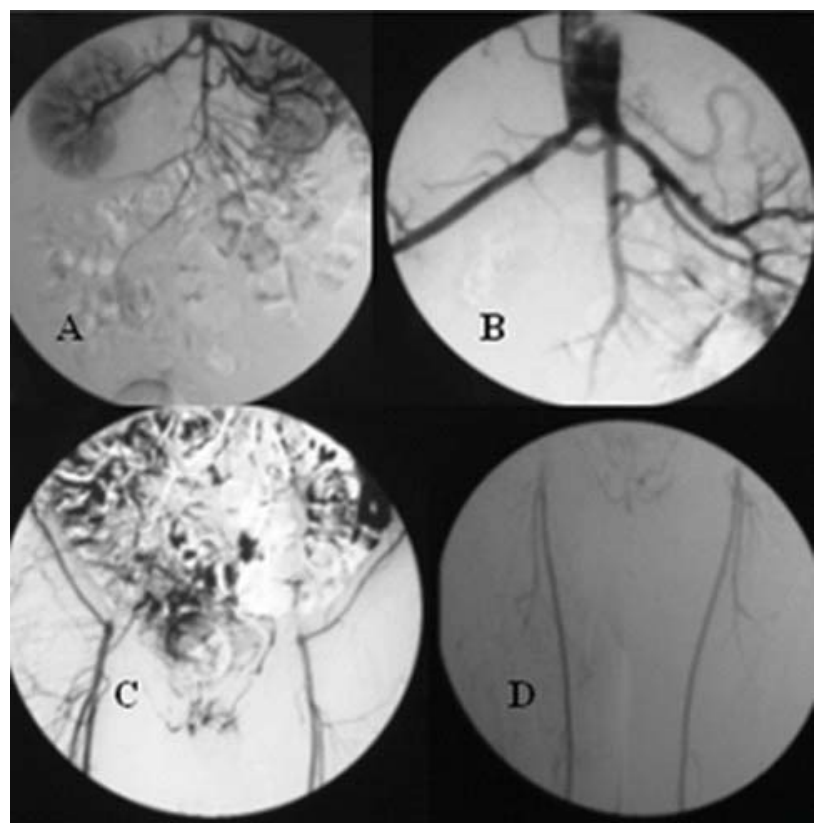

Figura 1 - A) Visão panorâmica da aortografia; B) detalhe da oclusão aórtica e visualização de ramos viscerais (renais e mesentérica superior); C) reenchimento das femorais comuns por colaterais; D) perviedade de femorais profundas, superficiais e transição fêmoro-poplítea

de gastrectomia há 16 anos devido a úlcera péptica e cirurgia de coluna lombar por acesso posterior há 7 anos.

Ao exame vascular, não se palpavam os pulsos femorais e distais. A angiografia digital mostrou oclusão da aorta abdominal imediatamente após os óstios das artérias renais e reenchimento das artérias femorais e poplíteas (Figura 1).

$\mathrm{O}$ paciente foi submetido a enxerto aorto-bifemoral utilizando-se prótese de Dacron 18 x $9 \mathrm{~mm}$. O acesso ao retroperitônio foi dificultado por aderências no andar supramesocólico do abdome atribuídas à cirurgia anterior. O paciente evoluiu sem intercorrências, e a dieta foi normalizada paulatinamente, recebendo alta no quinto dia pós-operatório (PO).

No $14^{\circ} \mathrm{PO}$, o paciente foi reinternado por queixa de vômitos e dor abdominal, porém mantinha a eliminação de flatos e fezes.

O paciente estava emagrecido, com abdome pouco distendido e difusamente doloroso à palpação, apresentava ruídos hidroaéreos normais e ausência de dor à descompressão brusca; as feridas operatórias permaneciam

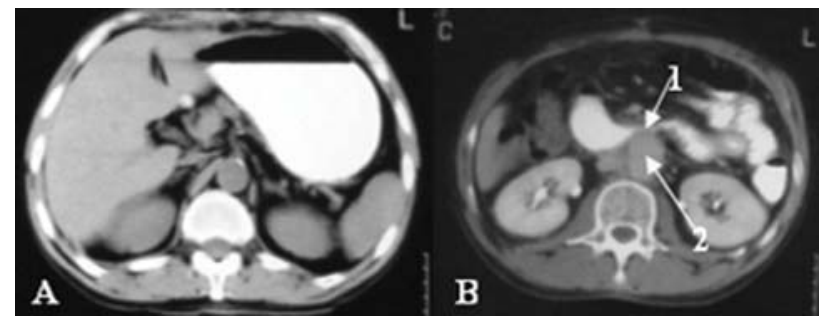

Figura 2 - A) Distensão gástrica; B) estenose duodenal (seta 1) e prótese de Dacron (seta 2)

em bom estado, e os pulsos distais dos membros inferiores, preservados. As hipóteses diagnósticas feitas foram bridas precoces, abscesso ou corpo estranho intracavitário e isquemia de cólon.

A retossigmoidoscopia não identificou nenhum sinal de isquemia colônica, porém a tomografia computadorizada mostrou distensão gástrica e imagem sugestiva de estenose da terceira porção duodenal (Figura 2).

Foram prescritos jejum, pró-cinéticos, sintomáticos e hidratação endovenosa. Uma sonda nasogástrica foi introduzida e deixada aberta.

No $23^{\circ} \mathrm{PO}$, foi realizada uma endoscopia digestiva alta durante a qual uma sonda nasoenteral foi introduzida até a transição da segunda para a terceira porção duodenal. Nessa topografia, observava-se "grande diminuição da luz, porém sem lesões".

A dieta enteral por sonda foi iniciada, e durante 3 dias o paciente se manteve sem vômitos; o abdome permanecia com som timpânico à percussão e doloroso após administração da dieta.

Optou-se pela reexploração cirúrgica no $27^{\circ} \mathrm{PO}$. A laparotomia revelou um pinçamento da terceira porção duodenal junto à sutura retroperitoneal.

Esse plano de sutura foi parcialmente desfeito; o retroperitônio, aberto; e a parede posterior da terceira porção duodenal e a prótese, expostas (Figura 3). A liberação do duodeno foi completada com a manobra de Kosher, e o trânsito duodenal foi restabelecido, conforme comprovou a endoscopia intra-operatória.

O retroperitônio foi selado com um remendo do grande omento, evitando-se, assim, novo pinçamento duodenal e protegendo-se a prótese. 


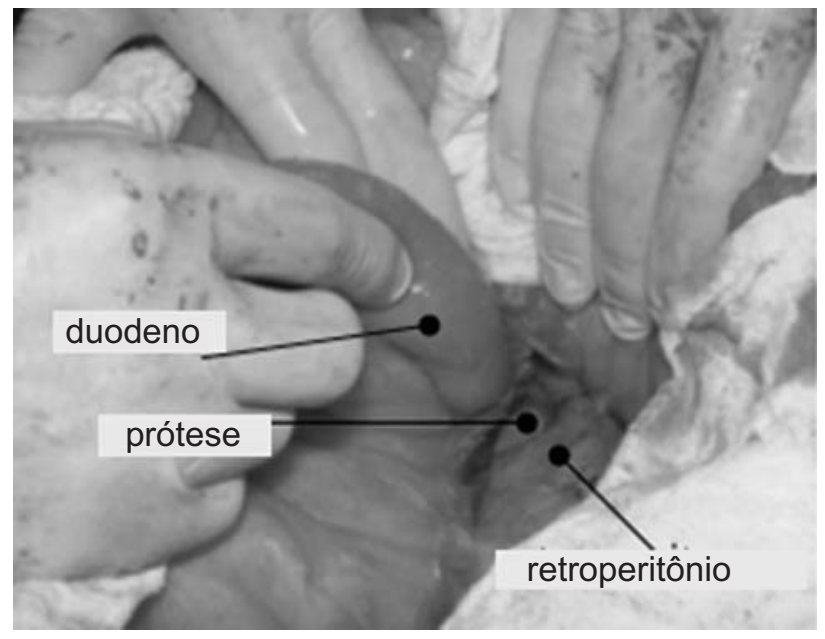

Figura 3 - Aspecto intra-operatório

O paciente foi mantido em jejum e com sonda nasogástrica até o terceiro PO da reexploração cirúrgica, quando apresentou ruídos hidroaéreos adequados. Foi iniciada dieta líquida.

No sexto PO, após progressão e aceitação da dieta, o paciente recebeu alta hospitalar. Manteve boa evolução após 6 meses de seguimento. Refere resolução da claudicação e da disfunção erétil, com os pulsos distais dos membros inferiores presentes e simétricos. Não ocorreram novas queixas digestivas.

\section{Discussão}

Embora complicações da cirurgia de aneurisma de aorta abdominal sejam bem descritas na literatura, existe uma carência de relatos relacionados à doença oclusiva aorto-ilíaca. A oclusão da prótese e o pseudo-aneurisma anastomótico são as principais complicações responsáveis pela necessidade de reintervenção ${ }^{3}$.

Complicações gastrointestinais após cirurgias da aorta feitas por via transperitoneal acontecem em 6,6 a $21 \%$ dos casos e estão associadas a grande morbidade e aumento da mortalidade ${ }^{1}$. O íleo paralítico é a mais comum; ocorre em 10\% dos casos. Hemorragia digestiva alta, enterocolite por Clostridium difficile e isquemia colônica também são freqüentes ${ }^{1}$.

O duodeno é o segmento do trato gastrointestinal que mais se relaciona aos problemas que envolvem a aorta abdominal. Sua situação retroperitoneal, praticamente repousando sobre a aorta, facilita sua implicação em várias situações, como na síndrome aortoduodenal, descrita em 1905 por Sir William Osler, na qual um aneurisma provoca obstrução do duodeno ao comprimi-lo de encontro à artéria mesentérica superior ou à própria parede abdominal ${ }^{4}$.

Outra complicação é a fístula aorto-entérica. Esta é a intercorrência mais grave relacionada ao trato digestivo, mas felizmente é rara e afeta menos de $1 \%$ dos pacientes $^{3,5,6}$. Um fator implicado na formação da fístula aorto-entérica é a cobertura inadequada do enxerto por tecido retroperitoneal. A aderência do enxerto à parede duodenal, a pulsatilidade excessiva de um enxerto redundante e a colonização da prótese por cepas de Staphylococcus epidermidis também são apontadas como fatores que contribuem para a formação de fistulas ${ }^{5}$.

Obstrução duodenal após cirurgia aórtica é uma complicação rara e ocorre em menos de $1 \%$ dos pacientes $^{2}$. Campagnol et al. cita apenas 11 casos de obstrução intestinal descritos até 1996 após correção de aneurisma ${ }^{1}$. Outro relato, mais recente, afirma que entre 1985 e 2004 apenas 18 casos foram descritos na literatura de língua inglesa ${ }^{2}$.

A obstrução em geral manifesta-se do oitavo ao $60^{\circ}$ PO (média de 13,3 dias) ${ }^{2}$. O quadro clínico pode incluir vômitos copiosos, algumas vezes biliosos, distensão abdominal, náuseas e dor abdominal ${ }^{2}$. O diagnóstico diferencial inclui íleo paralítico, hemorragia digestiva, neoplasia duodenal, pancreatite ${ }^{2}$, abscesso ou corpo estranho intracavitário e isquemia de cólon.

Entre os mecanismos de obstrução duodenal até então relatados estão aderências, síndrome da artéria mesentérica superior, coleção serosa no saco aneurismático, compressão por hematoma retroperitoneal ou da própria parede duodenal e fechamento incorreto do retroperitônio ${ }^{1,2}$. Um levantamento de 15 casos publicados entre 1966 e 1994 mostrou que dois pacientes foram operados por doença aórtica oclusiva, e 13, devido a aneurismas. Em 10 desses casos, o mecanismo mais comum de obstrução duodenal foi a formação aderências $^{1}$. Outros trabalhos apontam o hematoma retroperitoneal como a causa mais freqüente ${ }^{1}$. 
Cirurgias de doença oclusiva normalmente requerem exposição mais baixa da aorta, e por conseguinte há menor manipulação duodenal ${ }^{1}$. No caso relatado, como a oclusão aórtica era justa-renal, houve necessidade de dissecção acima das artérias renais. Além disso, nesse paciente, $\mathrm{o}$ acesso à aorta foi dificultado pelas bridas resultantes da cirurgia anterior (gastrectomia).

O tratamento da suboclusão duodenal no pósoperatório de procedimentos aórticos pode ser conservador ou cirúrgico ${ }^{2}$.

A obstrução precoce de alça do intestino delgado que requeira reintervenção cirúrgica, de modo geral, é acompanhada de uma mortalidade de $17 \%$ e aumento de morbidade provocado por abscessos intracavitários, infecção da ferida operatória, formação de fístulas, eventração e persistência da obstrução ${ }^{2}$.

A conduta conservadora, baseada no uso de descompressão gástrica, hidratação e sintomáticos, consegue evitar a reoperação em $73 \%$ dos casos. Quando a obstrução é supostamente devida a edema da parede duodenal, a corticoterapia endovenosa com betametasona $5 \mathrm{mg} /$ dia por 5 dias é uma opção ${ }^{1}$. Nutrição parenteral total também é válida quando o jejum é mantido por tempo prolongado ${ }^{2}$. Embora o período de tratamento possa variar de 7 a 66 dias, $83 \%$ dos pacientes tratados de forma conservadora têm o trânsito intestinal restabelecido em 2 semanas ${ }^{2}$.

A investigação do mecanismo responsável pela obstrução é essencial para conduzir o caso ${ }^{2}$. Exames complementares, como tomografia computadorizada, raios $\mathrm{X}$ contrastados do trato digestivo superior ${ }^{1,2}$ e endoscopia, podem ajudar a esclarecer qual o mecanismo envolvido em cada caso.

O tratamento conservador é efetivo na maior parte das obstruções causadas por compressões secundárias a hematomas retroperitoneais, intramurais ou por coleções do saco aneurismático ${ }^{2}$. Já as oclusões secundárias a aderências e ao fechamento inapropriado do retroperitônio raramente respondem às medidas clínicas ${ }^{2}$.

A técnica cirúrgica a ser utilizada vai depender de múltiplos fatores, como o mecanismo da obstrução, condições próprias dos órgãos envolvidos e alterações anatômicas provocadas por cirurgias prévias, entre outros.
Embora existam opções de derivações digestivas, como as gastrojejunais e duodenojejunais ${ }^{1}$, estas devem ser evitadas pelo risco de contaminação do enxerto aórtico, de deiscência de anastomoses e de formação de fístulas. Ocasionalmente, técnicas mais simples, como a evacuação de hematoma retroperitoneal, lise de bridas e remoção de uma sutura inadequada do retroperitônio, podem ser suficientes.

No caso da opção pela abertura do retroperitônio, esforços devem ser concentrados para que o enxerto aórtico não fique exposto, sobretudo sua anastomose proximal, com o intuito de dificultar a formação de fístulas aorto-entéricas. Pode ser feita nova síntese do retroperitônio, desde que haja tecido suficiente junto ao duodeno e em condições que permitam nova sutura. $\mathrm{O}$ uso de remendo do grande omento, interposto entre o duodeno e a prótese ${ }^{3,5,6}$, também é uma opção. Se essa tática não for possível, o omento pode ser substituído por materiais como a dura-máter ou pericárdio bovino.

Quando a síntese retroperitoneal é feita de modo habitual, o risco de fístulas aorto-entéricas é menor do que $1 \%$, e os sintomas podem surgir entre 8 e 180 meses após a colocação do enxerto, com média de 47 meses $^{4}$.

Não foram encontrados dados sobre a probabilidade de essa complicação se desenvolver quando o retroperitônio é fechado com a ajuda de remendos ou sobre quanto tempo levaria para se desenvolver. Também não há referências sobre protocolos de vigilância com exames complementares periódicos para tentar surpreender o surgimento da fístula aorto-entérica.

\section{Referências}

1. Campagnol M, Di Giacomo M, Cruciani R, Cavallaro A. Duodenal obstruction following elective abdominal aortic aneurysm repair. Cardiovasc Surg. 1996;4:843-5.

2. Lin CY, Lin BY, Kang PL. Duodenal obstruction after elective abdominal aortic aneurysm repair: a case report. Kaohsiung $\mathbf{J}$ Med Sci. 2004;20:501-5. 
3. Ruby BJ, Cogbill TH. Aortoduodenal fistula 5 years after endovascular abdominal aortic aneurysm repair with the Ancure stent graft. J Vasc Surg. 2007;45:834-6.

4. Adam DJ, Fitridge RA, Raptis S. Late reintervention for aortic graft-related events and new aortoiliac disease after open abdominal aortic aneurysm repair in an Australian population. J Vasc Surg. 2006;43:701-5; discussion 705-6.

5. Takagi H, Matsuno Y, Sekino S, Kato T, Umemoto T. Aortoduodenal syndrome. J Vasc Surg. 2006;43:851.
6. Armstrong PA, Back MR, Wilson JS, Shames ML, Johnson $\mathrm{BL}$, Bandyk DF. Improved outcomes in the recent management of secondary aortoenteric fistula. J Vasc Surg. 2005;42:660-6.

Correspondência:

Adenauer Góes Junior

Rua Dr. Pinto Ferraz, 271/123, Vila Mariana

CEP 04117-040 - São Paulo, SP

E-mail: adenauer-junior@ibest.com.br

\title{
ATENÇÃO
}

\section{Registros de Ensaios Clínicos}

O Jornal Vascular Brasileiro apóia as políticas para registro de ensaios clínicos da Organização Mundial da Saúde (OMS) e do International Committee of Medical Journal Editors (ICNJE, www.icmje.org), reconhecendo a importância dessas iniciativas para o registro e a divulgação internacional de informação sobre estudos clínicos, em acesso aberto. De acordo com essa recomendação, artigos de pesquisas clínicas devem ser registrados em um Registro de Ensaios Clínicos validados pelos critérios estabelecidos pela OMS e ICMJE, cujos endereços estão disponíveis no site do ICMJE:

\author{
www.actr.org.au \\ www.clinicaltrials.gov \\ www.isctn.org \\ www.umin.ac.jp/ctr/index/htm \\ www.trialregister.nl
}

O número de identificação deverá ser registrado ao final do resumo

A partir de 2008, somente serão aceitos para publicação artigos que tenham recebido um número de identificação nesses registros. 